\title{
Eficiência de tratamentos alternativos no controle de Pyricularia grisea em sementes de trigo
}

\author{
Cristina P. Silva ${ }^{1}$, Erika Nomura ${ }^{1}$, Edjane G. Freitas ${ }^{1}$, Caetano Brugnaro ${ }^{2}$ \& Alfredo S. Urashima ${ }^{1}$ \\ ${ }^{1}$ Departamento de Biotecnologia Vegetal; ${ }^{2}$ Departamento de Tecnologia Agroindustrial e Sócio-economia Rural, Centro de \\ Ciências Agrárias, Universidade Federal de São Carlos, 13600-970, Araras, SP, Brasil
}

Autor para correspondência: Alfredo S. Urashima, e-mail: alfredo@cca.ufscar.br

\begin{abstract}
RESUMO
O presente trabalho objetivou estudar métodos alternativos de controle de Pyricularia grisea, em sementes de trigo, uma das principais fontes de inóculo da brusone. Após ensaios preliminares para avaliação do efeito dos produtos, em diferentes concentrações, tempos e temperaturas de imersão sobre a germinação das sementes, os tratamentos hipoclorito de sódio $\left(2 \% ; \mathrm{pH} 11,5 ; 50^{\circ} \mathrm{C} ; 10 \mathrm{~min}\right) \mathrm{e}$ água quente $\left(55^{\circ} \mathrm{C} / 5 \mathrm{~min}\right)$ foram selecionados para estudar sua eficiência no controle de $P$. grisea e transmissão do patógeno para plântula. O delineamento estatístico constou de 400 sementes em 4 repetições em blocos ao acaso. A incidência do fungo foi examinada do $2^{\circ}$. ao $4^{\circ}$. dia através do "blotter test" a $22^{\circ} \mathrm{C}$ com fotoperíodo de $12 \mathrm{~h}$, a germinação das sementes foi avaliada aos 7 dias através da observação visual da emissão do tubo germinativo e a transmissão aos 7 e 14 dias pela identificação de conídios de $P$. grisea das lesões necróticas que surgiram em qualquer tecido da parte aérea. Dados do presente estudo revelaram que o tratamento hipoclorito de sódio $\left(\mathrm{pH} 11,5 / 50^{\circ} \mathrm{C} /\right.$ $10 \mathrm{~min}$ ) foi eficiente no controle de $P$. grisea em sementes de trigo pois reduziu a incidência inicial de $57,7 \%$ para $2,25 \%$, mas reduzindo significativamente a germinação para 46\%. O tratamento hipoclorito de sódio reduziu a transmissão do patógeno para plântulas de 39,7 para $0,5 \%$.
\end{abstract}

Palavras-chave: Magnaporthe grisea, Triticum aestivum, brusone, controle alternativo, sementes

\begin{abstract}
Efficiency of alternative treatments in the control of Pyricularia grisea in wheat seeds

The present study aimed to examine alternative methods of control of Pyricularia grisea in wheat seeds, one primary source of blast disease. The treatments sodium hypochlorite $(2 \% ; \mathrm{pH} 11,5 ; 50 \mathrm{C} ; 10 \mathrm{~min})$ and hot water $\left(55^{\circ} \mathrm{C} / 5 \mathrm{~min}\right)$ were selected after preliminary trials that examined the effect on germination of several reagents, at different concentrations, temperatures and period of submersion. Their efficiency in controlling $P$. grisea in wheat seeds and its transmission to seedlings was studied in seed lots of 400 in four replications in a randomized block design. The fungal incidence was analyzed through blotter test employing $22^{\circ} \mathrm{C}$ and photoperiod of $12 \mathrm{~h}$ from the second to fourth day, seed germination at the seventh day through visual emergence of germ tube and transmission to seedlings at the seventh and $14^{\text {th }}$ days through identification of $P$. grisea conidia from necrotic lesions of any aerial tissues. The present data revealed that sodium hypochlorite $\left(\mathrm{pH} 11.5 / 50^{\circ} \mathrm{C} / 10 \mathrm{~min}\right)$ was effective in controlling $P$. grisea in wheat seeds because it decreased the initial incidence from $57.7 \%$ to $2.25 \%$ but with deleterious effect on germination. This treatment reduced the fungal transmission to seedlings from 39.7 to $0.5 \%$.
\end{abstract}

Keywords: Magnaporthe grisea, Triticum aestivum, alternative control, seeds.

Brusone causada pelo fungo Pyricularia grisea (Cooke) Sacc. [teleomorfo Magnaporthe grisea (T.T. Hebert) M.E. Barr.] é uma das doenças mais destrutivas entre os fungos fitopatogênicos. Mais de 50 gramíneas são atacadas por esse patógeno, sendo a cultura do arroz seu hospedeiro mais importante (Ou, 1985). É de ocorrência generalizada em todas as regiões rizícolas do mundo, sendo considerada a principal doença da cultura em praticamente todas as regiões produtoras.

No Brasil, além do arroz, a brusone também foi constatada na cultura do trigo (Triticum aestivum) em 1985 (Igarashi et al., 1986), sendo esta a primeira observação no mundo da doença nessa cultura em condições naturais. O patógeno que inicialmente foi identificado em alguns municípios do norte do Paraná, rapidamente se disseminou para outras regiões tritícolas do país: São Paulo (Igarashi, 1990), Rio Grande do Sul (Picinini \& Fernandez, 1990), Mato Grosso do Sul (Goulart et al., 1990), Goiás (Prabhu et al., 1992), cerrado do Brasil Central (Anjos et al., 1996). Goulart \& Paiva (2000) observaram redução de 51\% do rendimento de grãos e incidência de $86 \%$ em campos de trigo no estado de Mato Grosso do Sul na safra 1991 e 1992 e mais recentemente Urashima et al. (2009) verificaram incidência de $76,2 \%$ e redução na produtividade de $32,2 \%$ na variedade BRS208 na região de Itapeva SP na safra de 2005. Urashima \& Kato (1994) verificaram que não existe nenhuma variedade de trigo resistente a todos os isolados do fungo. 
Uma das principais fontes de inóculo primário dessa doença é o plantio de sementes infectadas. Sementes infectadas foram consideradas a principal fonte de inóculo primário para as primeiras epidemias de brusone na Califórnia em 1997 (Greer \& Webster, 2001) e pela disseminação do fungo em trigo do Paraná para o Mato Grosso do Sul (Urashima et al., 1999).

Além disso, um outro fator que ressalta a importância de sementes como fonte de inóculo se reside no tempo de sobrevivência do fungo dentro desse órgão. P. grisea sobreviveu por mais de oito anos dentro de tecidos do pericarpo de milleto Eleusine coracana (Kato, 1978), por 22 meses em sementes de trigo em condições de laboratório (Reis et al., 1995) e foi encontrado em sementes de arroz preservados no banco de germoplasma por mais de 10 anos (Faiad et al., 1996), evidenciando que a viabilidade do fungo é igual ou maior que a da própria semente.

Os fatos mencionados acima destacam a importância de se incluir o tratamento de semente como uma das estratégias de controle da doença (Goulart \& Paiva, 1991, Pande et al., 1994). O tratamento de sementes com produtos químicos já foi bastante pesquisado e muitos fungicidas tem se mostrado eficientes (Lopes \& Bueno, 1990, Goulart \& Paiva, 1991). No entanto, a impossibilidade de uso em propriedades orgânicas, restrição ao uso de certos fungicidas no exterior e a crescente conscientização da população quanto à qualidade dos alimentos e do meio ambiente abrem a possibilidade de investigação de outros métodos de controle de patógenos em sementes. O objetivo do presente trabalho foi o de estudar diferentes tratamentos alternativos para o controle de P. grisea em sementes de trigo.

Amostras de sementes de trigo empregadas no estudo foram das cultivar BRS208 infectadas por P. grisea, coletadas no município de Itapeva em 2005 e se apresentavam enrugadas, de tamanho reduzido e de baixa qualidade, e estão preservadas em sílica gel em baixas temperaturas, no laboratório de Genética Molecular do CCA-UFSCar. Dados preliminares sobre efeito de diferentes produtos químicos em várias condições sobre a viabilidade das sementes de trigo, permitiram selecionar os tratamentos água quente $\left(55^{\circ} \mathrm{C} / 5 \mathrm{~min}\right)$ e $\mathrm{NaClO}(2 \% ; \mathrm{pH} 11,5 ; 50 \mathrm{C} ; 10 \mathrm{~min})$, para se examinar sua eficiência no controle de $P$. grisea e transmissão para plântulas empregando-se 400 sementes de trigo divididos em 4 repetições.

Asanidade das sementes foi examinada em laboratório de acordo com a metodologia utilizada por Goulart \& Paiva (1991) com modificações. Após cada tratamento, amostras de sementes foram colocadas em placa de petri e incubadas a $22^{\circ} \mathrm{C}$ fotoperíodo de $12 \mathrm{~h}$ por 2 a 4 dias. Após esse período as sementes foram avaliadas quanto a presença de $P$. grisea, através de microscópio estereoscópico. A germinação foi avaliada aos sete dias através da observação visual da emissão do tubo germinativo.

A transmissão do fungo para plântulas foi avaliada empregando-se os mesmos lotes. Essas sementes foram mantidas em placas de petri com papel de filtro umedecido a temperatura ambiente e fotoperíodo de $12 \mathrm{~h}$, sobre bancada de laboratório por um período de 14 dias. A avaliação da transmissão do patógeno foi realizada ao sétimo e $14^{\circ}$. dia, somente em plântulas que emergiram após serem submetidas aos tratamentos, através da identificação de conídias de $P$. grisea das lesões necróticas que surgiram em qualquer tecido da parte aérea, empregando-se fita adesiva e microscópio. $\mathrm{O}$ delineamento experimental foi o de blocos ao acaso com quatro repetições. A análise dos dados consistiu de Análise de Variância e Teste Tukey ao nível de $5 \%$ de probabilidade. Os dados de transmissão do teste final de eficiência dos produtos foram transformados em raiz quadrada antes da análise.

Hipoclorito de sódio nas condições mencionadas propiciou o melhor controle do patógeno, visto que a incidência reduziu de $57,7 \%$ para $2,25 \%$. Essa porcentagem foi estatisticamente diferente do tratamento com água quente que apresentou incidência de $11 \%$, que por sua vez se mostrou significativamente superior que a testemunha (Tabela 1). Esses valores diferentes de incidência do patógeno na semente proporcionaram diferentes níveis de transmissão dos sintomas nas plântulas, onde o melhor desempenho foi o do hipoclorito com somente $0,5 \%$ de transmissão, valor esse superior aos $12,2 \%$ de transmissão quando as sementes foram submetidas à água quente. No entanto, o hipoclorito de sódio teve um efeito deletério na germinação, pois sementes com germinação inicial de $84 \%$, apresentaram-se com $46 \%$ após o tratamento, diferença essa estatisticamente significativa.

Os dados evidenciaram que houve controle de P. grisea em sementes de trigo através do emprego de diferentes tratamentos alternativos, pois a incidência que se mostrava alto atingiu valores muito baixos, próximo ao zero. Dentre os produtos estudados, o que mais se destacou foi o hipoclorito de sódio que, quando empregado a $2 \%$ em $\mathrm{pH} 11,5$ e temperatura de $50^{\circ} \mathrm{C}$ por $10 \mathrm{~min}$, reduziu a incidência inicial de um lote de sementes de $57,7 \%$ para $2,2 \%$. Embora este decréscimo não tenha sido completo, o valor encontrado está abaixo dos níveis de tolerância para programas de certificação de sementes de trigo em relação a $P$. grisea, que é de 5\% para sementes básicas e de $10 \%$ para sementes certificadas (Machado, 1994).

Outro fator que reforça a eficiência do hipoclorito para controlar $P$. grisea foi que o controle foi obtido em lote de sementes de trigo que apresentavam infecção inicial superior a $57 \%$, um valor considerado alto pois levantamento da qualidade sanitária de sementes de trigo produzidas no Mato Grosso do Sul num período de 5 anos encontrou níveis de até $26,7 \%$, bem abaixo do empregado neste estudo (Goulart et al., 1995). Na eventualidade de se encontrar valores considerados altos estes lotes são empregados como grãos, sendo comercializados para moinhos e não como sementes. Pode-se aventar a hipótese de que tratamento termoterápico com hipoclorito de sódio em sementes com taxa menor de incidência, o controle proporcionado por este produto poderia ter sido melhor, fato já observado em sementes de brássicas (Babadoost et al., 1996). 
Eficiência de tratamentos alternativos no controle de Pyricularia grisea...

Tabela 1 - Efeito de hipoclorito de sódio e termoterapia na germinação e incidência de Pyricularia grisea em sementes de trigo e sua transmissão para plântulas

\begin{tabular}{lccc}
\hline \hline Tratamentos & Germinação (\%) & Incidência (\%) & Transmissão (\%) \\
\hline Testemunha & $84,00^{\mathrm{a}^{*}}$ & $57,75^{\mathrm{a}}$ & $39,75^{\mathrm{a}}$ \\
Água quente $\left(55^{\circ} \mathrm{C} / 5 \mathrm{~min}\right)$ & $75,25^{\mathrm{a}}$ & $11,00^{\mathrm{b}}$ & $12,25^{\mathrm{b}}$ \\
Hipoclorito de sódio $(2 \% ; \mathrm{pH} 11,5 ; 50 \mathrm{C} ; 10 \mathrm{~min})$ & $46,00^{\mathrm{b}}$ & $2,25^{\mathrm{c}}$ & $0,50^{\mathrm{c}}$ \\
C.V. $(\%)$ & 8,00 & 14,52 & 19,40 \\
\hline
\end{tabular}

*Médias seguidas de mesma letra na coluna não diferem estatisticamente entre si pelo teste Tukey, no nível de 5\% de probabilidade.

A eficiência do hipoclorito de sódio no controle de $P$. grisea em sementes de trigo teve desempenho equivalente aos melhores fungicidas químicos dos estudos de Goulart \& Paiva (1990) e Lopes \& Bueno (1990) demonstrando que controle alternativo de $P$. grisea em sementes deste cereal com este produto pode substituir fungicidas químicos em muitas situações. O hipoclorito de sódio já havia sido empregado com sucesso no controle de diferentes patógenos como os de pós-colheita Botrytis cinerea, Mucor piriformis e Penicillium expansum em peras (Spotts \& Peters, 1980), na germinação de patógenos da parte aérea, Tilletia indica, em trigo (Bonde et al., 1999), no tratamento de sementes de brássicas para Xanthomonas campestris $p v$. campestris (Babadoost et al., 1996), de arroz para bactérias e fungos (Chun et al., 1997), de ornamentais para Nimbya gomphrenae, Stemphylium vesicarium e Alternaria tagetica (Wu et al., 2001) e de tubérculos de batata para Rhizoctonia solani e Streptomyces scabies (Errampalli \& Johnston, 2001). Não há dados sobre o seu uso para sementes de trigo e nem seu efeito em P. grisea em outros hospedeiros como arroz e milhetos.

Existem fungicidas que em tratamento de sementes controlaram eficientemente $P$. grisea em trigo (Lopes \& Bueno, 1990; Goulart \& Paiva, 1991). A identificação de métodos alternativos que controlem $P$. grisea em sementes é interessante principalmente em propriedades orgânicas, onde o uso desses produtos não é permitido. Outra vantagem da não utilização do fungicida seria a baixa probabilidade de desenvolvimento de variantes resistentes, fato comum com o emprego de produtos químicos sistêmicos (Vincelli \& Dixon, 2002; Kim et al., 2003). Some-se a este fato a crescente preocupação da comunidade pelo uso excessivo de agrotóxicos, a contaminação ambiental e a qualidade dos alimentos produzidos, demonstrando o grande potencial de uso dessas alternativas no controle de $P$. grisea em sementes de trigo.

No entanto, para que o tratamento com esse reagente possa ser recomendado para o controle de $P$. grisea em sementes de trigo, existe a necessidade de estudos mais detalhados já que nas condições empregadas nesse trabalho, acarretou um efeito deletério na germinação, com redução significativamente menor que a testemunha. Esse ponto é um dos principais empecilhos para que o controle alternativo com termoterapia e produtos alternativos seja empregado em escala mais ampla, ou seja, a necessidade que existe de se realizar ensaios específicos para cada patossistema e cada lote de sementes. O emprego de $\mathrm{NaOCl}$ nas mesmas condições de concentração, $\mathrm{pH}$, temperatura e tempo de exposição em dois lotes de sementes diferentes proporcionou controles diferentes e diferença na taxa de germinação (dados preliminares não apresentados). Essa discrepância pode ser atribuído a diversos fatores como o menor número de sementes analisados no ensaio preliminar (dados não apresentados), mas existe a possibilidade que a diferença no controle tenha sido devido a diferenças da umidade das sementes, já que a umidade é um dos fatores primários para que a termoterapia seja bem sucedida pois a condução do calor para o interior da semente está diretamente ligado a umidade (Machado, 2000).

No presente trabalho, observou-se que existiu uma variação muito grande na germinação em função dos produtos e condições empregadas (dados preliminares não apresentados). Os dados aqui apresentados podem, assim, ser considerados preliminares mas indicativos da potencialidade do emprego do hipoclorito de sódio para o controle de P. grisea em sementes de trigo. Devido ao comprometimento da germinação existe a necessidade de pesquisas posteriores para que esse método de controle possa ser recomendado para uso em sementes de trigo.

Embora muitos dos tratamentos tenham proporcionado redução da incidência em sementes para valores abaixo de $10 \%$, estando portanto, dentro dos limites de tolerância para sementes certificadas (Machado, 1994), observou-se que a menor taxa de transmissão para plântulas foi no tratamento $\mathrm{NaClO}(2 \%$; $\mathrm{pH} 11,5 ; 50 \mathrm{C} ; 10 \mathrm{~min})$ onde sementes com incidência de 2,2\% tiveram transmissão de 0,5\% (Tabela 1). Esse dado é de extrema importância, com conseqüência epidemiologia muito grande, pois demonstra que mesmo com o emprego de sementes com incidência muito abaixo do padrão de tolerância de sementes básica e/ou certificadas, pode haver transmissão do patógeno para a parte aérea. Pelo fato de $P$. grisea ter explosiva capacidade esporulativa, com produção de novas gerações em menos de sete dias (Ou, 1985), tratamentos de sementes que não propiciem controle absoluto do patógeno nas sementes são temerários, pois o aparecimento de uma única lesão 
na parte aérea, advinda de semente pode se tornar fonte de inóculo primária da doença no campo e gerar epidemias de conseqüências desastrosas. Plantio de sementes infectadas foi a provável causa para a ocorrência da brusone em arroz em Arkansas (Long et al., 2001).

\section{AGRADECIMENTOS}

Os autores agradecem a Fundação de Amparo à Pesquisa do Estado de São Paulo - FAPESP pelo auxílio a pesquisa (04/14241-0) e a bolsa de iniciação científica FAPESP 05/52685-0 para o primeiro autor (05/52685-0).

\section{REFERÊNCIAS BIBLIOGRÁFICAS}

Anjos JRN, Da Silva DB, Charchar MJD, Rodrigues GC (1996) Ocorrência de brusone (Pyricularia grisea) em trigo e centeio na região dos cerrados do Brasil Central. Pesquisa Agropecuaria Brasileira 31:79-82.

Babadoost M, Derie ML, Gabrielson RL (1996) Efficacy of sodium hypochlorite treatments for control of Xanthomonas campestris pv.campestris in brassica seeds. Seed Science Technology 24:715.

Bonde MR, Nester SE (1999) Comparison of effects of acidic electrolyzed water and $\mathrm{NaOCl}$ on Tilletia indica teliospore germination. Plant Disease 83:627-632.

Chun SC, Schneider RW, Cohn MA (1997) Sodium hypochlorite: Effect of solution $\mathrm{pH}$ on rice seed disinfestations and its direct effect on seedling growth. Plant Disease 81:821-824.

Errampalli D, Johnston HW (2001) Control of tuber-borne black scurf (Rhizoctonia solani) and common scab (Streptomyces scabies) of potaoes with a combination of sodium hypochlorite and thiophanate-methyl preplanting seed tuber treatment. Canadian Journal Plant Pathology 23:68-77.

Faiad MGR, Wetzel MMVS, Salomão AN, Cunha R (1996) Evaluation of fungi in seed germplasm before long term storage. Seed Science Technology 24:505-511.

Goulart ACP, Paiva FA, Mesquita NA (1990) Ocorrência da brusone (Pyricularia oryzae) do trigo (Triticum aestivum) em Mato Grosso do Sul. Fitopatologia Brasileira 15:112-114.

Goulart ACP, Paiva FA (1991) Controle de Pyricularia oryzae e Helminthosporium sativum pelo tratamento de sementes de trigo com fungicidas. Pesquisa Agropecuária Brasileira 26:1983-1988.

Goulart ACP, Paiva FA (2000) Perdas no rendimento de grãos de trigo causadas por Pyricularia grisea, nos anos de 1991 e 1992, no Mato Grosso do Sul. Summa Phytopathologica 26:279-282.

Goulart ACP, Paiva FA, Andrade PJM (1995) Qualidade sanitária de sementes de trigo produzidos no Mato Grosso do Sul, safras 1987 a 1992. Summa Phytopathologia 21:235-238.

Greer CA, Webster RK (2001) Occurrence, distribution, epidemiology, cultivar reaction, and management of rice blast disease in California. Plant Disease 85:1096-1102.
Igarashi S (1990) Update on wheat blast (Pyricularia oryzae) in Brazil. In: Saunders, D.A. ed. Procceeding of the International Conference-Wheat for the nontraditional warm areas. 1990. Mexico, CIMMYT. pp. 480-483.

Igarashi S, Utiamada CM, Igarashi IC, Kazuma AH, Lopes RS (1986) Pyricularia em trigo. 1. Ocorrência de Pyricularia sp. no estado do Paraná. Fitopatologia Brasileira 11:351-352.

Kato H (1978) Biological and genetics aspects in the perfect state of rice blast fungus Pyricularia oryzae and its allies. Mutation breeding for disease resistance. Gamma field Symposium 17.

Kim YS, Dixon EW, Vincelli P, Farman ML (2003) Field resistance to strobilurin $\left(\mathrm{Q}_{\mathrm{O}} \mathrm{I}\right)$ fungicides in Pyricularia grisea caused by mutations in the mitochondrial cytochrome $b$ gene. Phytopathology 93:891-900.

Long DH, Correl JC, Lee FN, Tebeest DO (2001) Rice blast epidemics initiated by infested rice grain on the soil surface. Plant Disease 85:612-616.

Lopes MEBM, Bueno JT (1990) Estudos preliminares sobre detecção de Pyricularia sp em sementes de trigo (Triticum aestivum) e eficiencia do tratamento de sementes com funguicidas em condições de laboratorio. Summa Phytopathologica 16:166171.

Machado JC (1994) Padrões de tolerância de patógenos associados a sementes. Revisão Anual de Patologia de Plantas 2:229-263.

Ou SH (1985) Blast. In: Ou, SH (Ed.) $2^{\text {nd }}$ ed. Rice Diseases. Wallingford UK. CAB International. pp. 109-201.

Pande S, Mukuru SZ, Odhiambo RO, Karunakar RI (1994) Seedborne infection of Eleusine coracana by Bipolaris nodulosa and Pyricularia grisea in Uganda and Kenya. Plant Disease 78:6063.

Picinini EC, Fernandes JMC (1990) Ocorrência da brusone (Pyricularia oryzae) em lavouras comerciais de trigo (Triticum aestivum) no estado do Rio Grande do Sul. Fitopatologia Brasileira 15:83-84.

Prabhu AS, Filippi MC, Castro N (1992) Pathogenic variation among isolates of Pyricularia oryzae infecting rice, wheat, and grasses in Brazil. Tropical Pest Management 38:367-371.

Reis EM, Blum MC, Forcelini CA (1995) Sobrevivência de Pyricularia oryzae, associada as sementes de trigo. Summa Phytopathologica 21:43-44.

Spotts RA, Peters BB (1980) Chlorine and chlorine dioxide for control of d'Anjou pear decay. Plant Disease 64:1095-1097.

Urashima AS, Grosso CRF, Stabili A, Freitas EG, Silva CP, Netto DCS, Franco I, Mérola Bottan JH (2009) Effect of Magnaporthe grisea on seed germination, yield and quality of wheat. In: Wang GL, Valent B (Eds.) Advances in Genetics, Genomics and Control of Rice Blast Disease. Springer. pp. 267-277.

Urashima AS, Hashimoto Y, Don LD, Kusaba M, Tosa Y, Nakayashiki H, Mayama S (1999) Molecular analysis of the wheat blast population in Brazil with a homolog of retrotransposon MGR 583. Annals of the Phytopathological Society of Japan 65:429436.

Urashima AS, Kato H (1994) Varietal resistance and chemical control of wheat blast fungus. Summa Phytopathologica 20:107112.

Vincelli P, Dixon E (2002) Resistance to $\mathrm{Q}_{\mathrm{o}}$ ( (strobilurin-like) 
fungicides in isolates of Pyricularia grisea from perennial ryegrass. Plant Disease 86:235-240.

Wu WS, Chou HH, Lin SM, Wu HC (2001) The effect of seed- borne pathogens on emergence of globe amaranth, calendula and tagetes and the methods of control. Journal of Phytopathology 149:91-96.

Recebido 28 Fevereiro 2008 - Aceito 27 Fevereiro 2009 - TPP 8022

Editor Associado: Erlei M. Reis 